\title{
Der Vertrag von Lissabon - eine tragfähige und abschließende Antwort auf konstitutionelle Grundfragen?
}

\author{
Andreas Hofmann und Wolfgang Wessels*
}

\section{Ein Meilenstein der Integration}

Am 13. Dezember 2007 hat der Europäische Rat als konstitutioneller Architekt ${ }^{1}$ - nach mehrjährigem Vorlauf und mehr als halbjähriger konkreter Vorbereitungszeit - einen weiteren Meilenstein in der Geschichte der konstitutionellen Systemgestaltung der europäischen Integrationskonstruktion gesetzt. Ausgehend von der ,Berliner Erklärung ' vom 25. März $2007^{2}$ über die Verabschiedung eines Mandats für eine Regierungskonferenz ${ }^{3}$ gelang im Oktober die politische Einigung über einen ,Reformvertrag', der nun von den Staats- und Regierungschefs der Mitgliedstaaten in Lissabon unterzeichnet wurde. Das Dokument, das als „Vertrag von Lissabon“ in die Annalen der Integrationsgeschichte eingehen wird, ist somit als eine weitere Stufe in einem seit der Verabschiedung der Einheitlichen Europäischen Akte zwei Jahrzehnte währenden Prozess einzustufen, die Union ,demokratischer, transparenter und effizienter“4 zu gestalten - oder wie es jüngst die Staats- und Regierungschefs formulierten: „Um auch in Zukunft eine aktive Rolle in einer sich rasch verändernden Welt und im Hinblick auf die ständig wachsenden Herausforderungen spielen zu können, müssen wir die Handlungsfähigkeit der Europäischen Union und ihre Rechenschaftspflicht gegenüber dem Bürger bewahren und weiterentwickeln. " 5 Zu prüfen wird insbesondere sein, ob und wie nationale und europäische Politiker in der Zukunft das neue Regelwerk innerhalb oder auch neben den EU-Organen nutzen werden, um die selbst gesetzten Ziele zur Steigerung der Handlungsfähigkeit und demokratischen Kontrolle zu erreichen.

Das Dokument gewinnt eine besondere Bedeutung, da die Staats- und Regierungschefs der Mitgliedstaaten als Vertreter der „Herren der Verträge“6 einen ,Schwur“ auf die Bestän-

* Andreas Hofmann, M.A., Forschungsinstitut für Politische Wissenschaft und Europäische Fragen, Universität zu Köln.

Prof. Dr. rer. pol. Wolfgang Wessels, Jean Monnet Lehrstuhl, Forschungsinstitut für Politische Wissenschaft und Europäische Fragen, Universität zu Köln.

Die Autoren danken Wiebke Dreger und Niklas Helwig für vielfältige Zuarbeit.

1 Vgl. zu dieser Rolle des Europäischen Rats Wolfgang Wessels: Das politische System der Europäischen Union. Die institutionelle Architektur des EU-Systems, Wiesbaden 2008.

2 Erklärung anlässlich des 50. Jahrestages der Unterzeichnung der Römischen Verträge, 25.3.2007, abrufbar unter: http://www.eu2007.de/de/News/download_docs/Maerz/0324-RAA/German.pdf (letzter Zugriff: 21.12.2007). Vgl. Timo Goosmann: Die ,Berliner Erklärung ‘ - Dokument europäischer Identität oder pragmatischer Zwischenschritt zum Reformvertrag?, in: integration 3/2007, S. 251-263.

3 Europäischer Rat (Brüssel): Mandat für die Regierungskonferenz 2007, 23.06.2007, abrufbar unter: http://register.consilium.europa.eu/pdf/de/07/st11/st11218.de07.pdf (letzter Zugriff: 21.12.2007). Vgl. Klaus Hänsch: Ende gut - alles gut? Anmerkungen zum Reformvertrag, in: integration 4/2007, S. 499-502; Sylvie Goulard: Europäische Paradoxien - ein Kommentar zur Lage der EU, in: integration 4/2007, S. 503-511.

4 Europäischer Rat (Laeken): Erklärung von Laeken zur Zukunft der Europäischen Union, 15.12.2001, abrufbar unter: http://european-convention.eu.int/pdf/LKNDE.pdf (letzter Zugriff: 21.12.07). Vgl. u.a. Gerhard Brunn: Die Europäische Einigung, Stuttgart 2002; Jürgen Elvert: Die europäische Integration, Darmstadt 2006; Franz Knipping: Rom, 25. März 1957. Die Einigung Europas. 20 Tage im 20. Jahrhundert, München 2004.

5 Europäischer Rat (Brüssel): Schlussfolgerungen des Vorsitzes, 21./22. Juni 2007, abrufbar unter: http:// www.consilium.europa.eu/cms3_applications/Applications/newsRoom/LoadDocument.asp?directory=de/ec/ \&filename=94935.pdf (letzter Zugriff: 21.12.2007), hier Ziffer 2 .

6 Bundesverfassungsgericht: Urteil vom 12. Oktober 1993, BVerfGE 89,155, 190, „Maastricht“. 
digkeit des vorliegenden Werkes geleistet haben. So formuliert die Präambel des Lissabonner Vertrags ${ }^{7}$ den „Wunsch“, mit dem nun vorliegenden Vertragswerk ,den mit dem Vertrag von Amsterdam und dem Vertrag von Nizza eingeleiteten Prozess, mit dem die Effizienz und die demokratische Legitimität der Union erhöht und die Kohärenz ihres Handelns verbessert werden sollen, abzuschließen [eigene Hervorhebung]“. Die Schlussfolgerungen des Vorsitzes bestätigen diese Erwartung. Mit dem „Vertrag von Lissabon [erhalte] die Union einen stabilen und dauerhaften institutionellen Rahmen“. Sie erwarten ,,in absehbarer Zukunft keine weiteren Änderungen“" ${ }^{8}$ Bundeskanzlerin Angela Merkel bewertet den Lissabonner Vertrag ebenfalls als eine ,gelungene Neubegründung der Europäischen Union“. Die Legitimitätsfrage sei durch die Ausdehnung der Beteiligungsrechte sowohl nationaler Parlamente als auch des Europäischen Parlaments beantwortet, die Kompetenzordnung grenze die Zuständigkeiten der Union hinreichend ab und ein Rücktransfer von Kompetenzen an die Mitgliedstaaten sei nun rechtlich möglich. Schließlich führe die Einführung der doppelten Mehrheit sowohl zu gesteigerter Effizienz als auch - über das Bevölkerungskriterium zu legitimeren Entscheidungen. Anders als seine Vorgänger lasse dieser Vertrag ,keine Fragen offen".9

\section{Neue Struktur - weniger Komplexität?}

Der Vertrag von Lissabon nimmt einige grundlegende Änderungen an der bisherigen Struktur der vertraglichen Grundlagen des europäischen Konstrukts vor. Zwar behält der Vertrag von Lissabon vordergründig die Zweiteilung des Primärrechts bei, die Unterscheidung zwischen „Union“ und „Gemeinschaft“ wird jedoch aufgehoben. Der Ausdruck „Gemeinschaft" wird durchgängig durch den Ausdruck „Union“ ersetzt. Die vertragliche Grundlage der Union bilden von nun an der „Vertrag über die Europäische Union“ (EUV) sowie der „Vertrag über die Arbeitsweise der Europäischen Union“ (AEUV), der den bestehenden „Vertrag zur Gründung der Europäischen Gemeinschaft“ (EGV) ablöst.

Strukturelle Änderungen sind vor allem im EUV zu erkennen. Ein Blick auf die neu geordneten Titel lässt zunächst den Eindruck aufkommen, es handele sich hier nun um eine Schilderung der Grundlagen der Union, während der AEUV lediglich die Details der sektoralen Zusammenarbeit klärt (siehe Übersicht 1).

Bei näherer Betrachtung ist jedoch festzustellen, dass wichtige Elemente wie beispielsweise die - in dieser Form neue - Aufzählung der Zuständigkeiten der Union entgegen der augenscheinlichen Systematik im AEUV (Artikel 2 Absatz 6 AEUV) ${ }^{10}$ zu finden ist. Zudem fällt auf, dass sich in der Lissabonner Version des EUV kein Verweis mehr auf die ,polizeiliche und justizielle Zusammenarbeit in Strafsachen“ findet. Dieser Teil des Regelwerks wurde als neuer Titel V „Raum der Freiheit, der Sicherheit und des Rechts“ im Dritten Teil „Die internen Politiken und Maßnahmen der Union“ in den AEUV aufgenommen - die bisherige „dritte Säule“ der Union ist damit aufgelöst. Die Gemeinsame Außen- und Sicher-

7 Gemeint ist hier die Präambel des Änderungsvertrags, nicht der zu ändernden Verträge.

8 Europäischer Rat (Brüssel): Schlussfolgerungen des Vorsitzes, 14.12.2007, abrufbar unter: http://www.consilium.europa.eu/cms3_applications/Applications/newsRoom/LoadDocument.asp?directory=de/ec/\&filename=97683.pdf (letzter Zugriff: 21.12.2007), hier Ziffer 6 .

9 Regierungserklärung von Bundeskanzlerin Dr. Angela Merkel zur Unterzeichnung des Vertrages von Lissabon am 13. Dezember und zum Europäischen Rat am 14. Dezember 2007 vor dem Deutschen Bundestag am 12. Dezember 2007 in Berlin, abrufbar unter: http://www.bundesregierung.de/nn_1514/Content/DE/Bulletin/ 2007/12/141-1-bk-bt.html (letzter Zugriff: 21.12.2007).

10 Die Nummerierung der Vertragsartikel in diesem Beitrag bezieht sich auf die in der zukünftigen konsolidierten Version vorzunehmende Neunummerierung nach Artikel 5 Absatz 1 des Vertrags von Lissabon (entsprechend den Übereinstimmungstabellen im Anhang zu diesem Vertrag). 
heitspolitik (GASP) verbleibt jedoch als Titel V im EUV. In diesem Zusammenhang wird in Artikel 24 Absatz 1 EUV betont, dass für die GASP „besondere Verfahren“ gelten.

\section{Übersicht 1: Aufbau des Primärrechts entsprechend dem Vertrag von Lissabon}

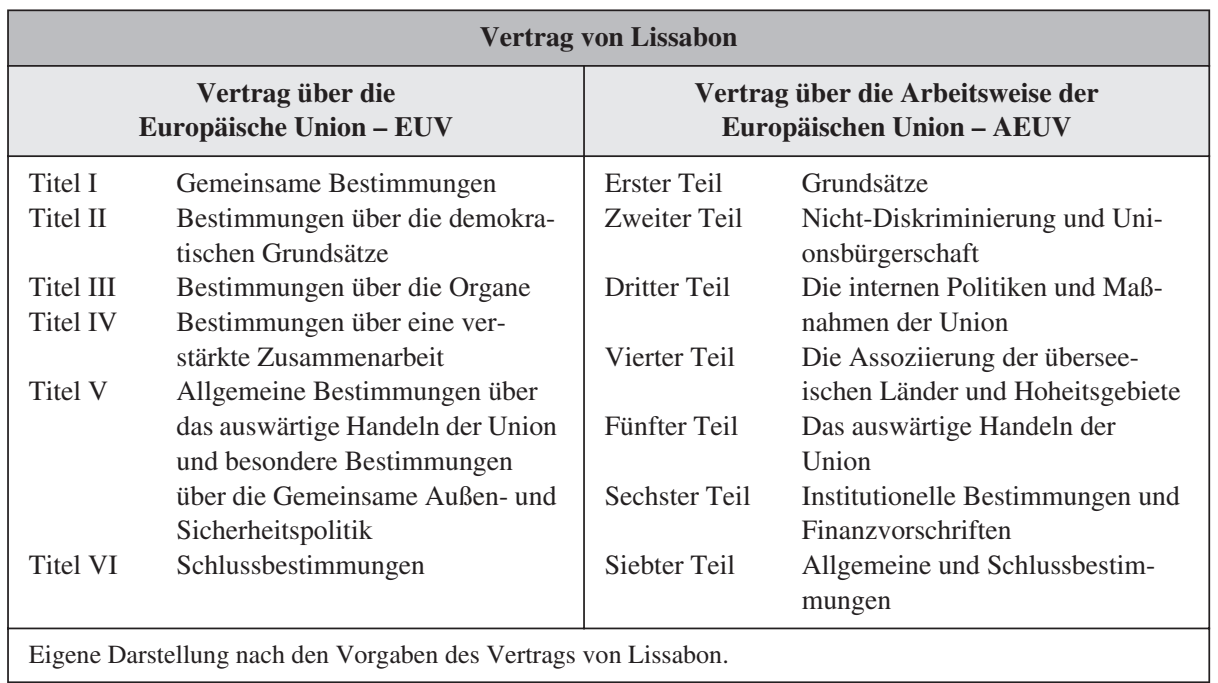

\section{Konstitutionelle Grundfragen: ein dreifaches Dilemma}

Für eine Einschätzung möglicher Wirkungen des Lissabonner Vertrags in der Realität der Union der 27 identifiziert unser Beitrag als Ausgangspunkt - in Anlehnung an die Zielsetzungen der Staats- und Regierungschefs - drei konstitutionelle Grundfragen in Hinblick auf die Gestaltung der Union:

1. Wie schreibt der Vertrag die Zuständigkeitsverteilung zwischen den Mitgliedstaaten und der Union und damit deren Aufgabenwahrnehmung fest?

2. Wie positioniert der Vertrag Akteure und strukturiert Verfahren zur Steigerung der Handlungsfähigkeit innerhalb der institutionellen Architektur?

3. Werden die neuen Vorschriften das EU-System ,demokratischer' gestalten und damit seine Legitimität steigern?

Unser Beitrag diskutiert die Antworten des Lissabonner Vertrags auf konstitutionelle Grundfragen, die jedes politische System beantworten muss. Als ein Ergebnis konstatieren wir sowohl strukturelle Dilemmata als auch ein Mehr an Handlungsfähigkeit und demokratischer Beteiligung. Dabei kommen wir - so bei der Betrachtung der Regelungen für Mehrheitsentscheidungen im Rat - zu Einsichten, die von bisher vorgelegten Analysen anderer Autoren abweichen. In einer Gesamtanalyse der institutionellen und prozeduralen Angebote des neuen Vertragswerks stellen wir zur Diskussion, wie unerprobte Veränderungen die institutionelle Architektur insgesamt beeinflussen und damit angesichts fortbestehender Spannungsverhältnisse Detailverbesserungen konterkarieren könnten.

Bei der Analyse der Kompetenz-, der institutionellen und der Verfahrensordnung des Lissabonner Vertrags sehen wir ein dreifaches Dilemma, das ambivalente Einstellungen und Verhaltensmuster der Mitgliedstaaten beim Ausbau des EU-Systems seit Beginn des Inte- 
grationsprozesses prägt und auch weiterhin wesentliche Elemente des Vertrags und dessen Komplexität in einem „Fusionsprozess" ${ }^{11}$ erklären hilft (siehe Übersicht 2).

\section{Übersicht 2: Das dreifache Dilemma}

\begin{tabular}{|l|l|l|}
\hline & \multicolumn{1}{|c|}{ Europäische Ebene } & \multicolumn{1}{c|}{ Nationale Ebene } \\
\hline Ebenendilemma & Problemlösungsinstinkt & Souveränitätsreflex \\
\hline Entscheidungsdilemma & Effizienzsuche & Letztentscheidungsvorbehalt \\
\hline Legitimitätsdilemma & eigenständig europäisch & abgeleitet national \\
\hline Eigene Darstellung. & & \\
\hline
\end{tabular}

Bei dem Dilemma der Aufgabenzuordnung unterstreichen die Regierungen der Mitgliedstaaten einerseits die Notwendigkeit, bei grenzüberschreitenden Problemen und globalen Herausforderungen in einer irgendwie gearteten Form gemeinsam ,zum Wohle der europäischen Bürger"12 vorgehen zu müssen. Sie betonen mit leicht variierenden Formulierungen immer wieder: „Europa ist geeint in der Überzeugung, dass wir in der Welt von morgen nur dann unsere Interessen und Ziele vertreten können, wenn wir zusammenarbeiten “. ${ }^{13}$ Deutlich tritt in diesen Formulierungen ein ,Problemlösungsinstinkt" der Mitgliedstaaten zu Tage, der zunehmend das EU-System als optimale „Problemlösungsebene“ erkennen lässt. ${ }^{14}$ Andererseits versuchen Bestimmungen des Vertrags von Lissabon in einem gegenläufigen ,Souveränitätsreflex ' noch stärker als zuvor die Zuständigkeiten der Union zu begrenzen, um das Risiko der Aushöhlung der de jure Souveränität der Mitgliedstaaten einzuschränken.

Nach der Entscheidung über die adäquate Problemlösungsebene stehen die Mitgliedstaaten bei der Wahl der Verfahren zur Beschlussfassung vor einem ,Entscheidungsdilemma'. Einerseits suchen die Mitgliedstaaten Verfahrensregeln, die die Entscheidungseffizienz stärken und damit „,die künftige Handlungsfähigkeit der Europäischen Union"15 sichern. Andererseits ist von Seiten der Mitgliedstaaten ein ausgeprägter Vorbehalt bei der Abgabe von Letztentscheidungsmöglichkeiten feststellbar, etwa in Form von Vetos im Rat.

Neben dem Prozess der Effizienzsteigerung in Kompetenzverteilung und Verfahrensordnung betont der Vertrag die Notwendigkeit, die demokratische Legitimität der Union zu steigern. Dieses Bestreben wird eindrücklich verdeutlicht durch die als Titel II neu in den EUV eingebrachten „Bestimmungen über die demokratischen Grundsätze“, die in Artikel 10 EUV eine eigenständig europäische und eine abgeleitete nationale Legitimationsdimension nebeneinanderstellen. Dieses Legitimitätsdilemma zeigt Wirkungen auf die Gestaltung des Texts, die durch rein effizienzsteigernde oder souveränitätserhaltende Motivationen alleine nicht erklärt werden können. Dabei ist zu beobachten, dass die Stärkung bestehender und die Einführung neuer Akteure zur Steigerung der Legitimität sich immer auch auf die Handlungsfähigkeit der Union auswirkt.

11 Vgl. Wolfgang Wessels: Die institutionelle Architektur des Verfassungsvertrags: Ein Meilenstein in der Integrationskonstruktion, in: Mathias Jopp/Saskia Matl (Hrsg.): Der Vertrag über eine Verfassung für Europa. Analysen zur Konstitutionalisierung der EU, Baden-Baden 2005, S. 45-85.

12 Europäischer Rat (Brüssel): Schlussfolgerungen des Vorsitzes, 21./22. Juni 2007, Ziffer 2.

13 Europäischer Rat (Brüssel): Schlussfolgerungen des Vorsitzes, 21./22. Juni 2007, Ziffer 1.

14 Vgl. Rudolf Hrbek/Wolfgang Wessels: Das EG-System als Problemlösungsebene und Handlungsrahmen, in: Rudolf Hrbek/Wolfgang Wessels (Hrsg.): EG-Mitgliedschaft: ein vitales Interesse der Bundesrepublik Deutschland? Bonn 1984, S. 501-542.

15 Europäischer Rat (Brüssel): Schlussfolgerungen des Vorsitzes, 21./22. Juni 2007, Ziffer 6. 


\section{Die Neuordnung der Kompetenzen: zwischen begrenzter Einzelermächtigung und staatsähnlicher Agenda}

\section{Ausprägungen des Souveränitätsreflexes}

Eine erste Durchsicht des Dokuments im Hinblick auf die Kompetenz- und Aufgabenzuordnung offenbart mehrere Ausprägungen des Souveränitätsreflexes. Der Lissabonner Vertrag übernimmt ausdrücklich nicht die quasi-konstitutionellen, staatsanalogisierenden Charakterisierungen des Verfassungsvertrags. Bereits im Mandat für die Regierungskonferenz wurde dekretiert, dass der EUV und der AEUV keinen „Verfassungscharakter“ haben dürfen. ${ }^{16}$ Die Artikel des Verfassungsvertrags zu den Symbolen der Union (Hymne, Flagge, Europatag, Wahlspruch) und Bezeichnungen wie „Europäisches Gesetz"17 sind im neuen Vertrag nicht zu finden. Auch die Position des „Außenministers der Union“ wird umbenannt in „Hoher Vertreter der Union für Außen- und Sicherheitspolitik“ und ähnelt damit der Ausschilderung dieser Position entsprechend dem gültigen Vertrag. Auch die Verbannung der Vorrangklausel des Unionsrechts gegenüber nationalem Recht vom Vertragstext in eine Erklärung ${ }^{18}$ ist als Ausdruck des Bemühens zu verstehen, einer unbegrenzten Kompetenzausweitung Grenzen zu setzen - auch wenn dieses aus der Rechtsprechung des Gerichtshofs hervorgehende Prinzip seit den frühen 1960er Jahren etabliert ist.

In diesem Sinne setzt auch der neu in den Vertrag aufgenommene systematisierende $\mathrm{Zu}$ ständigkeitskatalog in den „Gemeinsamen Bestimmungen“ des EUV und den „Grundsätzen“ des AEUV der Unionskompetenz deutliche Schranken auf. Die Grundsätze der Zuständigkeitsordnung (Artikel 4 und 5 EUV) betonen das Prinzip der „,begrenzten Einzelermächtigung“, die „Verhältnismäßigkeit“ und die „Subsidiarität“ und unterstreichen damit die Position der Mitgliedstaaten als „Herren der Kompetenzen“ ${ }^{19}$ Entsprechend achtet die Union die „nationale Identität“ der Mitgliedstaaten, ,die in ihren grundlegenden politischen und verfassungsmäßigen Strukturen einschließlich der regionalen und lokalen Selbstverwaltung zum Ausdruck kommt“ (Artikel 4 Absatz 2 EUV). Zudem definiert der Lissabonner Vertrag mit der „nationalen Sicherheit“ erstmals eine ausschließliche Zuständigkeit der Mitgliedstaaten (Artikel 4 Absatz 2 EUV). ${ }^{20}$ Das traditionelle Prinzip der ,,begrenzten Einzelermächtigung“ wird dadurch akzentuiert, dass ,alle der Union nicht in den Verträgen übertragenen Zuständigkeiten“ ausdrücklich bei den Mitgliedstaaten verbleiben (Artikel 4 Absatz 1 EUV). Artikel 48 Absatz 2 EUV betont zusätzlich, dass Regierungskonferenzen bei Änderungsverfahren des Vertrags auch Rückführungen beziehungsweise Verringerung von übertragenen Kompetenzen vorsehen können. Ein „,Protokoll über die Ausübung der geteilten Zuständigkeit“ grenzt zudem die Wirkungen geteilter Kompetenzen ein: „Die Ausübung von Zuständigkeiten [erstreckt] sich nur auf die durch den betreffenden Rechtsakt geregelten Elemente und nicht auf den gesamten Bereich." Schließlich sieht eine Erklärung im Bereich der geteilten Zuständigkeiten die Möglichkeit vor, einen Rechtsakt der Union aufzuheben und so Kompetenzen an die Mitgliedstaaten zurückzugeben. ${ }^{21}$ Auch Erklärungen zur GASP belegen den Souveränitätsreflex: So sollen Bestimmungen des Lissabonner

16 Europäischer Rat (Brüssel): Schlussfolgerungen des Vorsitzes, 21./22. Juni 2007, Ziffer 3.

17 Diese Bezeichnung lebt jedoch fort in den neu eingeführten ,ordentlichen“ und ,besonderen Gesetzgebungsverfahren".

18 Erklärung 17 zum Vorrang, Schlussakte zum Vertrag von Lissabon.

19 In Anlehnung an die „Herren der Verträge“; vgl. Bundesverfassungsgericht: Urteil vom 12. Oktober 1993, BVerfGE 89,155, 190, „Maastricht“.

20 Vgl. Peter-Christian Müller-Graff: Die Zukunft des europäischen Verfassungstopos und Primärrechts nach der deutschen Ratspräsidentschaft, in: integration 3/2007, S. 223-237.

21 Erklärung 19 zur Abgrenzung der Zuständigkeiten, Schlussakte zum Vertrag von Lissabon. 
Vertrags ,,weder die derzeit bestehenden Zuständigkeiten der Mitgliedstaaten für die Formulierung und Durchführung ihrer Außenpolitik noch ihre nationale Vertretung in Drittländern und internationalen Organisationen berühren". ${ }^{22}$ Ferner wird in Erklärung 14 festgestellt, dass im Vertrag von Lissabon ,der Kommission durch die Bestimmungen zur Gemeinsamen Außen- und Sicherheitspolitik keine neuen Befugnisse zur Einleitung von Beschlüssen übertragen werden und dass diese Bestimmungen die Rolle des Europäischen Parlaments nicht erweitern“. Expliziter als zuvor markiert der Vertrag Tabuzonen und ,domaines reservées“ der Mitgliedstaaten.

\section{Im Gegenzug: eine staatsähnliche Agenda?}

Im Gegensatz zu diesen anfänglichen Impressionen schreibt der Lissabonner Vertrag in der Präambel und den Gemeinsamen Bestimmungen des geänderten EUV einen breit angelegten Wertekatalog sowie eine umfangreiche Ziel- und Aufgabenliste fest. Im AEUV führen die Mitgliedstaaten - im augenscheinlichen Widerspruch zur verstärkten Grenzmarkierung und als Ausdruck des Problemlösungsinstinkts - einen umfassenden Kompetenzkatalog der Union in mehreren Abstufungen ein.

Deutlich wird aus den Formulierungen des Dokuments, dass die Vertragsväter und -mütter diese Union nicht als einen eng definierten Zweckverband ${ }^{23}$ einzig für die regulative Verwaltung eines gemeinsamen Binnenmarktes verstehen. Der neue Artikel 3 EUV spricht wesentliche Ziele staatlichen Handelns an; von allgemeinen Aufgaben der Förderung von Frieden, Werten und Wohlergehen ihrer Völker über (bereits an zweiter Stelle) den Raum der Freiheit, der Sicherheit und des Rechts, die Errichtung eines Binnenmarkts, sozial- und regionalpolitischen Aufgaben mit der Betonung von Solidarität, der kulturellen Vielfalt und des kulturellen Erbes Europas bis zur Wirtschafts- und Währungsunion, der Förderung der Werte der Union und des Schutzes der Unionsbürgerinnen und -bürger in den Beziehungen zur übrigen Welt.

Der mit dem Lissabonner Vertrag eingeführte Kompetenzkatalog teilt sich auf in ,ausschließliche“ (Zollunion, Wettbewerb, Währungspolitik, Fischerei, Handelspolitik) und ,geteilte" Zuständigkeiten (unter anderem Binnenmarkt, Sozialpolitik, Innen- und Justizpolitik, Landwirtschaft, Umwelt, Verbraucherschutz und Verkehr) sowie „Unterstützungs-, Koordinierungs- und Ergänzungsmaßnahmen“ der Union (unter anderem Gesundheit, Industrie, Kultur, Tourismus und Katastrophenschutz). Zusätzlich - quasi außerhalb dieses nachvollziehbaren Katalogs - bietet der Lissabonner Vertrag Möglichkeiten zur Koordinierung der Wirtschafts-, Beschäftigungs- und Sozialpolitik (Artikel 5 AEUV).

Zu dieser Liste zu zählen sind auch die im EUV (Artikel 24) aufgeführten Zuständigkeiten im Bereich der Gemeinsamen Außen- und Sicherheitspolitik und der Gemeinsamen Sicherheits- und Verteidigungspolitik (GSVP). ${ }^{24}$ Addiert man die erwähnten Politikfelder, so fehlt auf der EU-Ebene kein zentraler Bereich der nationalen Politik, auch wenn die Verfahren zu deren Ausgestaltung in ihrer Vielfalt keine durchgängige Zentralisierung erwarten lassen.

Grundsätzlich sieht der Lissabonner Vertrag nun eine durchgängige Rechtsbasis vor, die durch die Verleihung der „Rechtspersönlichkeit“ (Artikel 47 EUV) nochmals unterstrichen wird. Ziel dabei ist es, eine durchgängige Systematik bei den „Arten von Zuständigkeiten der Union“ (Artikel 2 AEUV) und bei den „Rechtsakten der Union“ (Artikel 288 AEUV) zu erreichen.

22 Erklärung 13 zur Gemeinsamen Außen- und Sicherheitspolitik, Schlussakte zum Vertrag von Lissabon.

23 Hans-Peter Ipsen: Europäisches Gemeinschaftsrecht, Tübingen 1972.

24 Interessanterweise schildert der Lisabonner Vertrag diesen Bereich jetzt als „Gemeinsam“ und nicht mehr als „Europäisch“ aus. 
Im Hinblick auf den Problemlösungsinstinkt ist schließlich auf Flexibilitätsoptionen in der Zuständigkeitsverteilung hinzuweisen, die ihren Ausdruck in Artikel 352 AEUV als Ermächtigung zur Vertragslückenschließung finden, jedoch in spezifischer Form auch an anderen Stellen im Vertrag zu finden sind. ${ }^{25}$

\section{Legitimität durch eigenständig europäische Grundrechte?}

Trotz ihrer Verbannung aus dem Vertragswerk sind zum Kapitel der Kompetenzordnung auch der Artikel zur Charta der Grundrechte und das dazugehörende Protokoll zu zählen. Zwar wird im neuen Artikel 6 Absatz 1 EUV auf die Charta Bezug genommen und ihr ,dieselbe Rechtsverbindlichkeit wie die Verträge“ zugesprochen, der Text selber wird jedoch nicht Teil des Primärrechts werden. In diesem Kontext werden die Auswirkungen des Legitimitätsdrucks deutlich: Aufgrund ihrer bereits erreichten Kompetenzfülle soll die Union einerseits als Wertegemeinschaft den Grundrechtsschutz auf europäischer Ebene kodifizieren und die Charta der Grundrechte in einer modifizierten Fassung als rechtsverbindlich anerkennen. Dem Souveränitätsreflex folgend betont der zweite Unterabsatz des Artikel 6 Absatz 1 EUV jedoch: „Durch die Bestimmungen der Charta werden die in den Verträgen festgelegten Zuständigkeiten der Union in keiner Weise erweitert".

Für die Anwendung der Charta sieht nun ein zusätzliches Protokoll26 eine Sonderregelung für das Vereinigte Königreich und Polen ${ }^{27}$ vor. Dieses schafft eine weitere Form von ,Opt-outs ' in der ohnehin langen Liste von Ausnahmeregelungen, die insbesondere das Vereinigte Königreich in Anspruch nimmt. Bestanden bisherige Varianten insbesondere darin, bei geplanten neuen Politikfeldern nicht mitzumachen, wird mit diesem Protokoll eine Ausnahmeregel vom Grundsatz gleicher Rechte und Pflichten formuliert. Die Einheitlichkeit der Rechtsgemeinschaft, die als ein besonderes Gut der Integrationskonstruktion gesehen wird, wird dadurch erneut und verstärkt durchbrochen. ${ }^{28}$

\section{Die Neuordnung der institutionellen Architektur: Legitimitätsdruck und Handlungs- fähigkeit}

\section{Demokratische Grundsätze: duale Legitimität als institutionelle Leitidee}

Angesichts einer breiten, wenn auch zwiespältigen Aufgabenzuordnung an die UnionsEbene setzt sich das Dilemma der Mitgliedstaaten beim Ausbau der institutionellen Architektur auf europäischer Ebene fort. Der Vertrag von Lissabon setzt so - gleichsam als institutionelle Leitidee - den veränderten „Bestimmungen über die Organe“ die ebenfalls neuen „Bestimmungen über die demokratischen Grundsätze“ der Union voran. Diese Bestimmungen lassen ein duales Legitimationsprinzip erkennen: die Union als ,Union der Bürgerinnen und Bürger" und als ,Union der Staaten'. Die Beteiligung der Bürger auf der europäischen Ebene als eigenständig europäisches Legitimationselement wird mit ihrer direkten Vertretung durch das Europäische Parlament gewährt, während die Mitgliedstaaten durch ihre de-

25 Vgl. Rudolf Streinz: Europarecht, 6. Auflage, Heidelberg 2003, S. 191.

26 Protokoll über die Anwendung der Charta der Grundrechte der Europäischen Union auf Polen und das Vereinigte Königreich.

27 Polen wird auch unter der neuen Regierung von Donald Tusk am Opt-out von der Grundrechte-Charta festhalten. Dieser Schritt gilt als Kompromiss gegenüber Staatpräsident Lech Kaczyński, der andernfalls mit einem Veto gedroht hatte.

28 Vgl. Walter Hallstein: Die Europäische Gemeinschaft, 5. Auflage, Düsseldorf/Wien 1979, S. 56-61; Franz C. Mayer: Europa als Rechtsgemeinschaft, in: Gunnar Folke Schuppert/Ingolf Pernice/Ulrich Haltern (Hrsg.): Europawissenschaften, Baden-Baden 2005, S. 429-488. 
mokratisch legitimierten Regierungen im Rat und im Europäischen Rat vertreten sind (Artikel 10 Absatz 2 EUV).

Als zentrale Grundsätze dieser Leitidee können „Gleichheit“ (Artikel 9 EUV), ,repräsentative Demokratie“ (Artikel 10 EUV Absatz 1) und „Partizipation“ (Artikel 11 EUV) identifiziert werden. Die „demokratischen Grundsätze“ umfassen auch den Verweis auf die Rolle politischer Parteien in der Herausbildung eines „europäischen politischen Bewusstseins“ (Artikel 10 Absatz 4 EUV) sowie die Rolle der nationalen Parlamente (Artikel 12 EUV).

\section{Akteure in der institutionellen Architektur: auf dem Weg zu einem neuen institutionellen} Gleichgewicht?

Der Lissabonner Vertrag sieht eine Reihe von Veränderungen im institutionellen Aufbau der Union vor, deren Formulierungen einen hohen Grad an Mehrdeutigkeit signalisieren und damit auch potenzielle Konflikte für die Handlungsfähigkeit der Union erkennen lassen. ${ }^{29}$

Eine prominente Neuerung ist der (Vollzeit-)Präsident des Europäischen Rats, der nun für eine Amtszeit von zweieinhalb Jahren gewählt wird (Artikel 15 Absatz 5 EUV). Seine Aufgabenbeschreibung lässt die Präferenz der Staats- und Regierungschefs erkennen, ihre eigene Institution handlungsfähiger zu gestalten, legt aber auch den Schluss nahe, dass die Vertreter der „Herren der Verträge“ eine zusätzliche Instanz zur Kontrolle anderer Institutionen installieren wollen. ${ }^{30}$

Schließt man das ,Gesetz der unerwarteten Folgen“ nicht aus, so kann diese Position wie in der öffentlichen Debatte bereits immer wieder anklingt - auch zum ,Präsidenten der Union' insgesamt stilisiert werden. Die Rolle des Präsidenten des Europäischen Rats kann so zwischen zwei Polen verortet werden: der Amtsinhaber kann als ein ,üblicher' Vorsitz handeln, der die Arbeit seines Gremiums vereinfacht und beschleunigt; er kann sich aber auch als Präsident im Sinne der französischen Rollendefinition verstehen, der insbesondere nach außen als Repräsentant ,Europas' auftritt. ${ }^{31}$

Eine Innovation besonderer Art ist der „Hohe Vertreter der Union für Außen- und Sicherheitspolitik“ (Artikel 18 EUV), der der Union mit der Unterstützung eines „Europäischen Auswärtigen Diensts“ (Artikel 27 Absatz 3 EUV) in der internationalen Politik ,Gesicht“ und ,Stimme“ verleihen soll. Der Hohe Vertreter wird dazu den Vorsitz im Rat für „Auswärtige Angelegenheiten“ einnehmen, gleichzeitig übernimmt er die Funktion des derzeitigen Kommissars für Außenbeziehungen und fungiert dabei als einer der Vizepräsidenten der Kommission (Artikel 18 Absätze 3 und 4 EUV). Dieser ,Doppelhut', den der Amtsträger tragen soll, ist ein fast schon idealtypischer Indikator des Ebenen- und Entscheidungsdilemmas, aus dem heraus die Herren der Verträge eine komplexe institutionelle Fusion vorgenommen haben. Zum Schutz gegen eine, ungebührliche' Stärkung dieser Position wird gleichzeitig die weiterhin bestehende außen- und sicherheitspolitische Autonomie der Mitgliedstaaten betont, ${ }^{32}$ die sich auch im Festschreiben intergouvernementaler Verfahren niederschlägt - mit Einstimmigkeit im Rat als Regelfall, einer schwachen Position für Kom-

29 Vgl. zur intensiven Diskussion u.a. CEPS/EGMONT/EPC: The Treaty of Lisbon. Implementing the Institutional Innovations, Brüssel 2007, S. 7; Christian Lequesne: Towards a new institutional balance?, in: EPC (Hrsg.): The people's project? The new EU Treaty and the prospects for future integration, Brüssel 2007, S. $10-16$.

30 Vgl. u.a. Hussein Kassim/Anand Menon: The Principal-Agent Approach and the Study of the European Union, in: Journal of European Public Policy 1/2003, S. 121-139.

31 Vgl. CEPS/EGMONT/EPC: The Treaty of Lisbon, 2007, S. 49.

32 Erklärung 13 zur Gemeinsamen Außen- und Sicherheitspolitik, Schlussakte zum Vertrag von Lissabon. 
mission und Parlament und ohne Zuständigkeit für den Gerichtshof (Artikel 24 Absatz 1 EUV). Die Wahrnehmung dieses Amts wird so höchst schwierig werden. ${ }^{33}$

Gegenüber diesen neuen Ämtern stärkt der Lissabonner Vertrag aber auch die Rechte des Präsidenten der Kommission (Artikel 17 Absatz 6 EUV) sowie dessen Legitimation durch die „Wahl“ seitens des Europäischen Parlaments (Artikel 14 Absatz 1 EUV). ${ }^{34}$ Eine ab 2014 einsetzende Verkleinerung der Kommission soll zudem die Handlungsfähigkeit dieser Institution verbessern.

Bei der unausweichlichen Auseinandersetzung um zentrale Führungsrollen darf der Ratsvorsitz als vierte Institution in diesem neu geschaffenen ,Führungsquartett " nicht übersehen werden. Im Rat bleibt das bisherige Rotationsprinzip mit einigen Änderungen bestehen. So soll eine Gruppe von jeweils drei Mitgliedstaaten eine ,Team-Präsidentschaft' für einen Zeitraum von 18 Monaten wahrnehmen. ${ }^{35}$ Die Verteilung soll nach dem Prinzip der gleichberechtigten Rotation unter den Mitgliedstaaten ,unter Berücksichtigung ihrer Verschiedenheit und des geografischen Gleichgewichts innerhalb der Union“ festgelegt werden.

Wurde mit Bezug auf die gegenwärtige Regelung in der kurzen Dauer des Vorsitzes ein Defizit hinsichtlich der Kontinuität der Zielsetzung über den Zeitraum des Vorsitzes hinaus identifiziert, stellt sich nun die Frage der Koordination zwischen den diversen Vorsitzen. ${ }^{36}$ Dies gilt nicht zuletzt im Hinblick auf die Vorbereitung und Durchführung der Tagungen des Europäischen Rats, an der sowohl der Präsident desselben als auch der Kommissionspräsident als Mitglieder sowie der Hohe Vertreter als Teilnehmer (Artikel 15 Absatz 2 EUV) und die Team-Präsidentschaft über den Vorsitz des Rats für „Allgemeine Angelegenheiten“ (Artikel 16 Absatz 2 EUV) beteiligt sind. ${ }^{37}$

Durch die Aufstellung des neuen Führungsquartetts wird die Aufgabenwahrnehmung zwar auf eine kontinuierlichere Basis gestellt, aber die Vertragsbestimmungen erhöhen gleichzeitig die Zahl der Akteure in Verantwortungspositionen. Nachteile bilden zumindest in den ersten Jahren die zu erwartende Verwirrung bezüglich Zuständigkeiten, Wettstreit um Einfluss und damit eine Verwässerung und Verwischung von Verantwortlichkeiten. Die Stärkung der einzelnen Positionen kann in der Summe zu einem ungeordneten Neben- und Gegeneinander innerhalb der institutionellen Architektur führen und so die Handlungsfähigkeit - entgegen den Absichten der Vertragsväter und -mütter - schwächen.

\section{Die Neuordnung der Verfahren: Schritte zum Ausbau einer effizienten und legitimen (Gemeinschafts-)Methode}

\section{Mehr europäische Demokratie? Ausbau der Beteiligungsrechte des Europäischen Parla- ments und die Bürgerinitiative}

Deutlich feststellbar ist eine Ausweitung von Verfahrensmodalitäten, die in der Regel der Gemeinschaftsmethode zugeschrieben werden. Wie die vergangenen Vertragsrevisionen wird auch der Lissabonner Vertrag die Aufgaben des Europäischen Parlaments ausbauen, dessen parlamentarische Funktionen stärken und somit die eigenständig europäische Legitimitätsdi-

33 Vgl. Graham Avery: The new architecture for EU foreign policy, in: EPC (Hrsg.): The people's project?, 2007, S. $17-25$.

34 Vgl. CEPS/EGMONT/EPC: The Treaty of Lisbon, 2007, S. 27.

35 Erklärung 9 zu Artikel 9c Absatz 9 des Vertrags über die Europäische Union betreffend den Beschluss des Europäischen Rates über die Ausübung des Vorsitzes im Rat, Schlussakte zum Vertrag von Lissabon.

36 Vgl. CEPS/EGMONT/EPC: The Treaty of Lisbon, 2007, S. 41-46.

37 Vgl. u.a. Philippe de Schoutheete: The European Council, in: John Peterson/Michael Shackleton (Hrsg.): The Institutions of the European Union, Oxford 2002, S. 21-46. 
mension der Union verstärken. Von besonderer Bedeutung ist in diesem Zusammenhang der Ausbau der Legislativrechte des Parlaments (siehe Abbildung 1). Das bisherige Mitentscheidungsverfahren, das dem Europäischen Parlament dem Rat gleichgestellte Beteiligungsmöglichkeiten einräumt, wird nicht nur dem Begriff nach zum „ordentlichen Gesetzgebungsverfahren“, sondern auch der Zahl der Artikel nach zu einem ,Normalfall‘. Der Lissabonner Vertrag wird dieses Verfahren in 35 Entscheidungsfällen zusätzlich einführen und auf weitere zentrale Politikbereiche ausdehnen - so auf die Vorschriften zur Asyl- und Einwanderungspolitik sowie auf die Maßnahmen im Kampf gegen internationale Kriminalität und Terrorismus. ${ }^{38}$ Es stellt so unter den Vertragsartikeln das mit Abstand am häufigsten anzuwendende einzelne Verfahren dar (in 80 von 256 Vorgaben mit Verfahrensbezug).

Abbildung 1: Entwicklung vertraglicher Beteiligungsrechte des Europäischen Parlaments

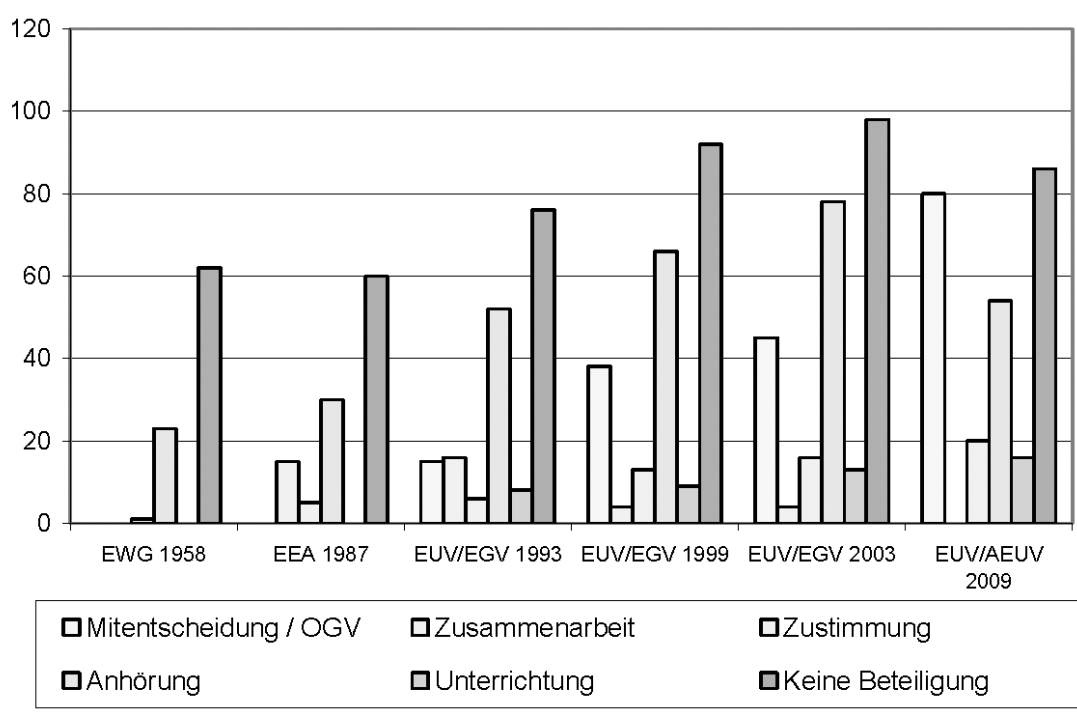

OGV = Ordentliches Gesetzgebungsverfahren

Quelle: eigene Darstellung aufbauend auf Wessels: Das politische System der Europäischen Union, 2008.39

Neben diesem Verfahren gewinnt im geschriebenen Text auch das Verfahren der Zustimmung an weiterer Bedeutung für Grundsatzentscheidungen. Auch im jährlichen Haushaltsverfahren, das der Lissabonner Vertrag - im Unterschied zu den gegenwärtig gültigen Vertragsartikeln - in wesentlichen Schritten dem ordentlichen Gesetzgebungsverfahren annähert, hat das Parlament an Rechten gewonnen.

Bezüglich der Wahlfunktion hat der Vertrag von Lissabon eindeutig die Rechte des Europäischen Parlaments festgeschrieben. Es „,wählt den Präsidenten der Kommission“ (Artikel

38 Vgl. CEPS/EGMONT/EPC: The Treaty of Lisbon, 2007, S. 7.

39 Die Berechnungen für den EUV/AEUV 2009 beruhen auf der inoffiziellen konsolidierten Version der Unionsverträge, zusammengestellt von Peadar ó Broin vom Irish Institute of European Affairs (IIEA), abrufbar unter: http://www.iiea.com/images/managed/publications_attachments/consolidated_treaties_draft.pdf (letzter Zugriff: 21.12 .2007$)$. 
14 Absatz 1 EUV) mit der „Mehrheit seiner Mitglieder“ (Artikel 17 Absatz 7 EUV), auch wenn das Initiativrecht für dieses Verfahren beim Europäischen Rat bleibt.

Auch im Bereich der Vertragsrevisionen weitet der Vertrag von Lissabon die bisher sehr spärlichen Beteiligungsrechte des Parlaments aus und richtet nun auch rechtlich gefasste Möglichkeiten zur Initiative und zur Vorbereitung von Regierungskonferenzen im Bereich des ordentlichen und des vereinfachten Änderungsverfahrens ein (Artikel 48 EUV). Durch seine Mitwirkung im einzuberufenden Konvent im Rahmen des ordentlichen Änderungsverfahrens ist das Parlament nun zusätzlich an der Ausformung der Vertragsänderungen beteiligt. Die endgültige Beschlussfassung in den Änderungsverfahren ist jedoch allein den Mitgliedstaaten und damit de facto dem Europäischen Rat vorbehalten. Der Lissabonner Vertrag bestätigt damit das Monopol der Herren der Verträge - und damit den Souveränitätsreflex - bei diesem Letztentscheidungsrecht über Kompetenzen und Verfahrensordnung der Union.

Neben dem Ausbau der Beteiligungsrechte des Europäischen Parlaments ist auch die Einführung einer „Bürgerinitiative“ zur Verbesserung - eigenständig europäischer - demokratischer Legitimität näher zu diskutieren. Artikel 11 Absatz 4 EUV erlaubt einer Anzahl von mindestens einer Million Unionsbürgern ,,aus einer erheblichen Anzahl von Mitgliedstaaten“ die Kommission aufzufordern, Rechtsakte zur Umsetzung der Verträge zu initiieren. Obwohl diese Bestimmungen also eine Option direkter Partizipation schaffen, erscheint die Tragweite dieses direktdemokratischen Elements zunächst begrenzt. Die Kommission bleibt das Nadelöhr; ihr Initiativmonopol wird auch durch Bürgerbeteiligung nicht umgangen. Es erscheint jedoch möglich, dass derartige Referenden - eventuell stärker als politische Parteien im Europäischen Parlament - zur Herausbildung eines ,europäischen politischen Bewusstseins“ und zum „Ausdruck des Willens der Bürgerinnen und Bürger der Union“ (Artikel 10 Absatz 4 EUV) beitragen können.

\section{Mehr Handlungsfähigkeit? Ausdehnung der Mehrheitsabstimmungen im Rat}

Für die Entscheidungsmodalitäten des Rats bleibt zunächst festzuhalten, dass die Mitgliedstaaten im Lissabonner Vertrag die Anwendungsbereiche für eine Beschlussfassung nach den Regeln der qualifizierten Mehrheit im Rat gegenüber der Version von Nizza auf 21 neue und 23 bestehende Politikbereiche ausdehnen (siehe Abbildung 2). ${ }^{40}$ Unter den von dieser Ausweitung erfassten Politikbereichen ist insbesondere die Innen- und Justizpolitik zu nennen. Hier stellt nun das qualifizierte Mehrheitswahlverfahren den Regelfall. ${ }^{41}$

Als Teil des Souveränitätsreflexes und des Letztentscheidungsvorbehalts haben die Mitgliedstaaten jedoch in zentralen Bereichen der Innen- und Justizpolitik (Artikel 82 und 83 AEUV) und der Sozialpolitik (Artikel 48 AEUV), für die laut Lissabonner Vertrag ein Mehrheitsvotum vorgesehen wird, Vetomöglichkeiten eingeführt.

Im Falle der Bedrohung ,wichtiger Aspekte [des] Systems der sozialen Sicherheit“ (im Bereich der Sozialpolitik) oder ,grundlegender Aspekte [der] Strafrechtsordnung“ (im Bereich der Innen- und Justizpolitik) kann ein Mitgliedstaat die Suspendierung des Mehrheitswahlverfahrens und eine Überweisung an den Europäischen Rat verlangen, der einstimmig entscheidet. Im Bereich der GASP wird eine solche ,Notbremse " fortgeschrieben (Artikel 31 Absatz 2 EUV).

40 CEPS/EGMONT/EPC: The Treaty of Lisbon, 2007, S. 73.

41 In den Bestimmungen des Lissabonner Vertrags lassen sich in diesem Politikbereich 18 Anwendungsbereiche des qualifizierten Mehrheitswahlverfahrens gegenüber 10 der Einstimmigkeit identifizieren. 
Abbildung 2: Entwicklung der Entscheidungsarten im Rat 1952-2007

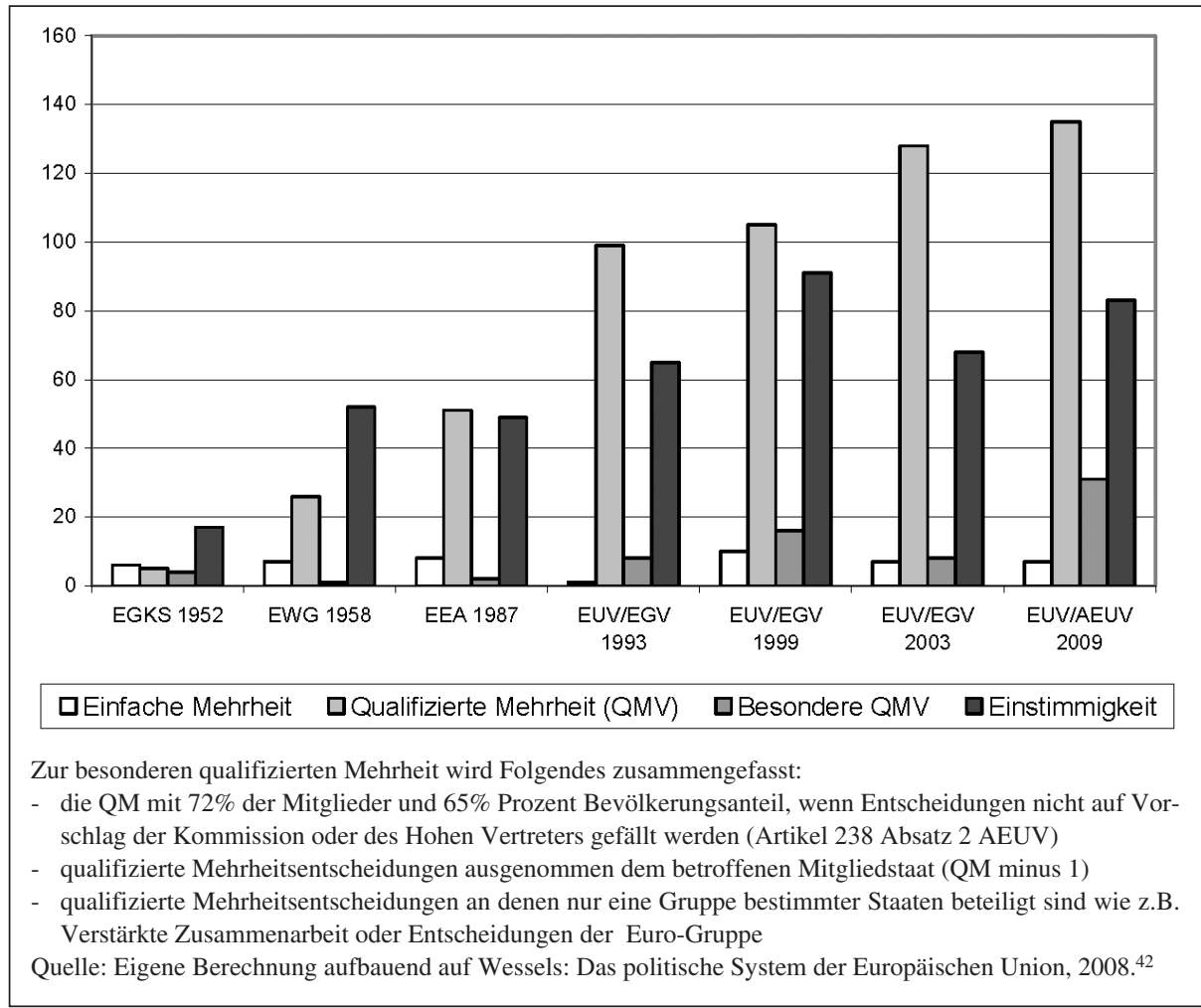

Die geschilderten ,Notbremsen` erfahren ihrerseits wiederum Einschränkungen. Der Einspruch einzelner Mitgliedstaaten in den genannten Bereichen kann demnach - so in der Innen- und Justizpolitik - von anderen Mitgliedstaaten zur Einleitung einer ,verstärkten Zusammenarbeit" genutzt werden. ,Notbremse" und ,Gashebel' sind so eng verknüpft.

Zusätzlich bietet der Vertrag von Lissabon durch sogenannte Brückenregelungen („Passerelle-Klauseln") die Möglichkeit, eine Effizienzsteigerung der Verfahren durch die Einführung von Mehrheitsentscheidungen in Bereichen zu erreichen, in denen laut Vertrag zunächst Einstimmigkeit vorgesehen ist (Artikel 48 EUV).

Einschränkungen und Variationen des Letztentscheidungsvorbehalts? Die Regelungen zur qualifizierten Mehrheit im Rat

Während bei der Ausweitung der Anwendungsbereiche des qualifizierten Mehrheitswahlverfahrens im Unterschied zu vorangegangenen Regierungskonferenzen wenige Kontroversen beobachtbar waren, erwiesen sich die Regeln für Mehrheitsabstimmungen im Rat als zentrale Kontroverse im Vorfeld der politischen Einigung zum Lissabonner Vertrag.

42 Die Berechnungen für den EUV/AEUV 2009 beruhen auf der inoffiziellen konsolidierten Version der Unionsverträge, zusammengestellt von Peadar ó Broin. 
Im Zentrum der Auseinandersetzung stand die Suche nach einem Ausgleich zwischen Effizienzsuche in der Beschlussfähigkeit des Organs und der Absicherung nationaler Letztentscheidungsvorbehalte.

Nach zähem Ringen einigte man sich letztlich auf eine Übernahme der, doppelten Mehrheit', die die Bestimmungen des Verfassungsvertrags aufgreift. ${ }^{43}$ Als Kompromiss wurde jedoch beschlossen, die Einführung der doppelten Mehrheit bis zum 1. November $2014 \mathrm{zu}$ verzögern (Artikel 16 Absatz 4 EUV). Bis dahin gelten die Bestimmungen des Vertrags von Nizza mitsamt seinen gewichteten Stimmen und dem dreifachen Quorum (siehe Übersicht 3).

Für einen Übergangszeitraum vom 1. November 2014 bis zum 31. März 2017 räumt das „Protokoll über die Übergangsbestimmungen“ zudem die Möglichkeit ein, auch nach Einführung der doppelten Mehrheit auf Antrag eines Mitgliedstaates weiterhin auf die Regelungen von Nizza zu rekurrieren. Damit besteht für diesen Zeitraum von zweieinhalb Jahren eine Art ,Reserveoption".

Die doppelte Mehrheit setzt für das Zustandekommen eines Rechtsakts die Zustimmung von mindestens 55 Prozent der Mitglieder des Rats voraus, gebildet aus mindestens 15 Mitgliedern, ${ }^{44}$ sofern die von diesen vertretenen Mitgliedstaaten zusammen mindestens 65 Prozent der Bevölkerung der Union ausmachen (Artikel 16 Absatz 4 EUV - siehe Übersicht 3). Als eine Konzession an die bevölkerungsarmen Mitgliedstaaten setzt der zweite Unterabsatz voraus, dass für die Bildung einer Sperrminorität mindestens vier Mitglieder des Rates (das heißt also nicht nur drei große Mitgliedstaaten, die zusammen mehr als 35 Prozent der Bevölkerung repräsentieren) notwendig sind, andernfalls gilt die qualifizierte Mehrheit als erreicht. Dieses letzte Kriterium verleitet einige Kommentatoren dazu, hier eher von einer ,dreifachen Mehrheit“ zu sprechen. ${ }^{45}$

Analog zu den bestehenden Regelungen gilt zudem ein erhöhtes Quorum für diejenigen Rechtsakte, die nicht auf Vorschlag der Kommission oder des Hohen Vertreters zustande kommen. In diesen in 13 Vertragsartikeln vorgesehenen Fällen ist eine Zustimmung von 72 Prozent der Mitgliedstaaten bei gleichbleibendem Bevölkerungsquorum für eine Verabschiedung des Rechtsakts vonnöten.

\section{Übersicht 3: Bestimmungen über die qualifizierte Mehrheit nach Nizza und Lissabon}

\begin{tabular}{|l|l|}
\hline \multicolumn{1}{|c|}{ Vertrag von Nizza (EU 27) } & \multicolumn{1}{c|}{ Vertrag von Lissabon } \\
\hline - Mehrheit der Mitgliedstaaten & - 55\% der Mitgliedstaaten (mind. 15) \\
- 255 der insgesamt 345 gewogenen Stimmen & - 65\% der Bevölkerung \\
(ca. 74\%) & - Sperrminorität: mind. 4 Mitgliedstaaten \\
- 62\% der Gesamtbevölkerung der Union (Prü- \\
fung auf Antrag eines Mitgliedstaats)
\end{tabular}

43 Vgl. Wessels: Die institutionelle Architektur, in: Jopp/Matl: Der Vertrag über eine Verfassung für Europa, 2005.

44 Dieser eigentlich überflüssige Zusatz ist wohl der Verhinderung mathematischer Spitzfindigkeiten geschuldet. Relevant könnte er wohl im Falle eines nun legal möglichen Austritts eines Mitgliedstaates werden.

45 CEPS/EGMONT/EPC: The Treaty of Lisbon, 2007, S. 63. 
Zur Ermöglichung eines Kompromisses sieht der Vertrag von Lissabon - insbesondere auf Drängen der polnischen Regierung - zudem eine Erklärung ${ }^{46}$ vor, die den sogenannten Kompromiss von Ioannina ${ }^{47}$ aufgreift und die Möglichkeit einer Form des ,suspensiven Vetos ‘ eröffnet. Nach dieser Formel können Mitglieder des Rates für die Übergangsperiode vom 1. November 2014 bis zum 31. März 2017 den Rat zu weitergehenden Verhandlungen auffordern, falls sie mindestens drei Viertel der Bevölkerung oder mindestens drei Viertel der Mitgliedstaaten vertreten, die für die Bildung einer Sperrminorität erforderlich sind. Der Rat soll ,alles in seiner Macht Stehende“ tun, um innerhalb einer ,angemessenen Zeit" und unter Beachtung der vertraglich vorgesehenen zwingenden Fristen eine zufriedenstellende Lösung zu finden. ${ }^{48}$ Als Kompensation für den Wegfall der ,Reserveoption ‘ ab dem 1. April 2017 soll der Schwellenwert ab diesem Zeitpunkt dauerhaft auf 55 Prozent der Mitgliedstaaten beziehungsweise 55 Prozent der Bevölkerung, die für die Bildung einer Sperrminorität erforderlich sind, gesenkt werden. Übersicht 4 präsentiert einen Überblick über die Kriterien der qualifizierten Mehrheit, die im Falle der Ratifikation des Lissabonner Vertrags sukzessive in Kraft treten werden und fasst den Ablauf der Regeländerungen zusammen.

\section{Übersicht 4: Vertragsregelungen zur Bestimmung der qualifizierten Mehrheit}

\begin{tabular}{|c|c|}
\hline Zeitablauf & Vertragsregelung \\
\hline Gegenwärtig & Berechnung nach den Regelungen von Nizza (Artikel 205 EGV) \\
\hline 1. Jan. 2009 & $\begin{array}{l}\text { Berechnung nach den Regelungen von Nizza (Protokoll über die Übergangsbestim- } \\
\text { mungen, Artikel } 3 \text { Absatz 3) }\end{array}$ \\
\hline \multirow[t]{3}{*}{ 1. Nov. 2014} & Berechnung nach der doppelten Mehrheit von Lissabon (Artikel 16 Absatz 4 EUV) \\
\hline & $\begin{array}{l}\text { Auf Antrag eines Mitgliedstaats: Berechnung nach den Regelungen von Nizza (Pro- } \\
\text { tokoll über die Übergangsbestimmungen, Artikel } 3 \text { Absatz 2) }\end{array}$ \\
\hline & $\begin{array}{l}\text { Suspensives Veto (Beschluss des Rates über die Anwendung des Artikels } 16 \text { Absatz } 4 \\
\text { EUV, Artikel 1): } \\
\text { - } 33,75 \% \text { der Mitgliedstaaten (drei Viertel der Sperrminorität): } 10 \text { Mitgliedstaaten, } \\
\text { oder } \\
\text { - } 26,25 \% \text { der Bevölkerung (drei Viertel der Sperrminorität): ca. } 128 \text { Mio. }\end{array}$ \\
\hline \multirow[t]{2}{*}{ 1. April 2017} & Berechnung nach der doppelten Mehrheit von Lissabon (Artikel 16 Absatz 4 EUV) \\
\hline & $\begin{array}{l}\text { Suspensives Veto (Beschluss des Rates über die Anwendung des Artikels } 16 \text { Absatz } 4 \\
\text { EUV, Artikel 4): } \\
\text { - } 24,75 \% \text { der Mitgliedstaaten ( } 55 \% \text { der Sperrminorität): } 7 \text { Mitgliedstaaten, oder } \\
\text { - } 19,25 \% \text { der Bevölkerung ( } 55 \% \text { der Sperrminorität): ca. } 94 \text { Mio. }\end{array}$ \\
\hline
\end{tabular}

Bei der Bewertung dieser Regelungen ist eine gewisse Nüchternheit geboten. Der verbesserten Handlungsfähigkeit des Rates (und damit der Union insgesamt) kommt in erster Linie

46 Erklärung 7 zu Artikel 16 Absatz 4 des Vertrags über die Europäische Union und zu Artikel 238 Absatz 2 des Vertrags über die Arbeitsweise der Europäischen Union, Schlussakte zum Vertrag von Lissabon.

47 Beschluss des Rates vom 29. März 1994 über die Beschlussfassung des Rates mit qualifizierter Mehrheit. Vgl. u.a. CEPS/EGMONT/EPC: The Treaty of Lisbon, 2007, S. 62.

48 Erklärung 7 zu Artikel 16 Absatz 4 des Vertrags über die Europäische Union und zu Artikel 238 Absatz 2 des Vertrags über die Arbeitsweise der Europäischen Union, Schlussakte zum Vertrag von Lissabon. 
die Ausweitung der Anwendungsfälle der qualifizierten Mehrheit zugute. Dieses Entscheidungsverfahren - ungeachtet seiner spezifischen Regelungen - setzt die Verhandlungen im Rat einer Dynamik aus, die die Beschlussfassung erleichtert. ${ }^{49}$ In diesen Politikfeldern kann sich kein Mitgliedstaat darauf verlassen, seine Interessen durch Ausübung eines Vetorechts zu wahren.

Zunächst ist festzustellen, dass die qualifizierte Mehrheitswahl ein normales Phänomen im Alltag des Rats darstellt, also vom Rat tatsächlich praktiziert wird. Entgegen der häufig geäußerten Darstellung, Konsens stelle im Rat die ,, angemessene “50 Verhaltensnorm dar und verdränge daher die Relevanz von vertraglich vorgesehenen Mehrheitsentscheidungen, zeigen empirische Studien die Akzeptanz der qualifizierten Mehrheitswahl. Von 2002 bis 2006 wurde demnach in 10 bis 22 Prozent der Rechtsakte des Rats tatsächlich abgestimmt. ${ }^{51}$ Die Verhaltensmuster von Ministern und Beamten mögen zwar eine deutliche Neigung zur Konsenssuche zeigen, sie werden jedoch durch das Risiko einer möglichen Überstimmung dynamisiert: Verhandlungen im Rat stehen - bei entsprechenden Vertragsregeln - nach Aussagen von beteiligten Akteuren immer im "Schatten“ möglicher Abstimmungen. ${ }^{52}$

Die Einschätzung über die Auswirkungen der neuen Bestimmungen auf die Handlungsfähigkeit des Rats variieren. Manche Analysen sehen im Senken der Schwelle für Mehrheitsbeschlüsse einen Gewinn an Entscheidungseffizienz, rechtlicher Sicherheit und demokratischer Verantwortlichkeit; auch statistische Wahrscheinlichkeitsrechnungen prognostizieren eine deutliche Steigerung der Beschlusseffizienz des Rates nach den Regelungen von Lissabon. ${ }^{53}$ Dagegen lässt eine hier bevorzugte Auflistung möglicher Koalitionen für gestaltende Mehrheiten beziehungsweise für Sperrminoritäten keine wesentliche Verbesserung der Handlungsfähigkeit des Rats erkennen (siehe Übersicht 5). Lediglich geringfügige Veränderungen gegenüber dem gegenwärtig gültigen Regelwerk sind ersichtlich, so etwa der Verlust der Sperrminorität für die 2004/2007 beigetretenen Mitgliedstaaten und für die Ostseeanrainer. Auch weiterhin stellen die 23 kleinsten Mitgliedstaaten keine gestaltende Mehrheit, die größten 14 verlieren zudem aufgrund des neuen Staatenquorums ihre Gestaltungsmehrheit, obwohl sie 90 Prozent der Unionsbevölkerung repräsentieren. Im Gegensatz zu anderen Darstellungen ${ }^{54}$ geben die neuen Regelungen demnach weder dem Bevölkerungsquorum noch dem Staatskriterium ein Übergewicht. Angesichts dieser Ergebnisse ist auch die häufig vorgenommene Analyse der Veränderungen in der jeweiligen relativen Entscheidungsmacht einzelner Staaten weniger bedeutsam. ${ }^{55}$

49 Vgl. u.a. Fiona Hayes-Renshaw/Helen Wallace: The Council of Ministers, New York 2006; CEPS/EGMONT/ EPC: The Treaty of Lisbon, 2007, S. 66.

50 Vgl. James G. March/Johan P. Olsen: The logic of appropriateness, ARENA Working Papers 04/09, Oslo 2004.

51 Vgl. Sara Hagemann/Julia De Clerck-Sachsse: Old Rules, New Game. Decision-Making in the Council of Ministers after the 2004 Enlargement, Brüssel 2007, S. 13.

52 Vgl. Hayes-Renshaw/Wallace: The Council of Ministers, 2006, S. 259.

53 Vgl. CEPS/EGMONT/EPC: The Treaty of Lisbon, 2007, S. 66; Richard Baldwin/Mika Widgrén: Council voting in the Constitutional Treaty: Devil in the details, CEPS Policy Brief Nr. 53/2004.

54 Vgl. CEPS/EGMONT/EPC: The Treaty of Lisbon, 2007, S. 66.

55 Vgl zu einer näheren Analyse CEPS/EGMONT/EPC: The Treaty of Lisbon, 2007, S. 67-72. 
Übersicht 5: Koalitionen für qualifizierte Mehrheiten und Sperrminoritäten (EU 27)

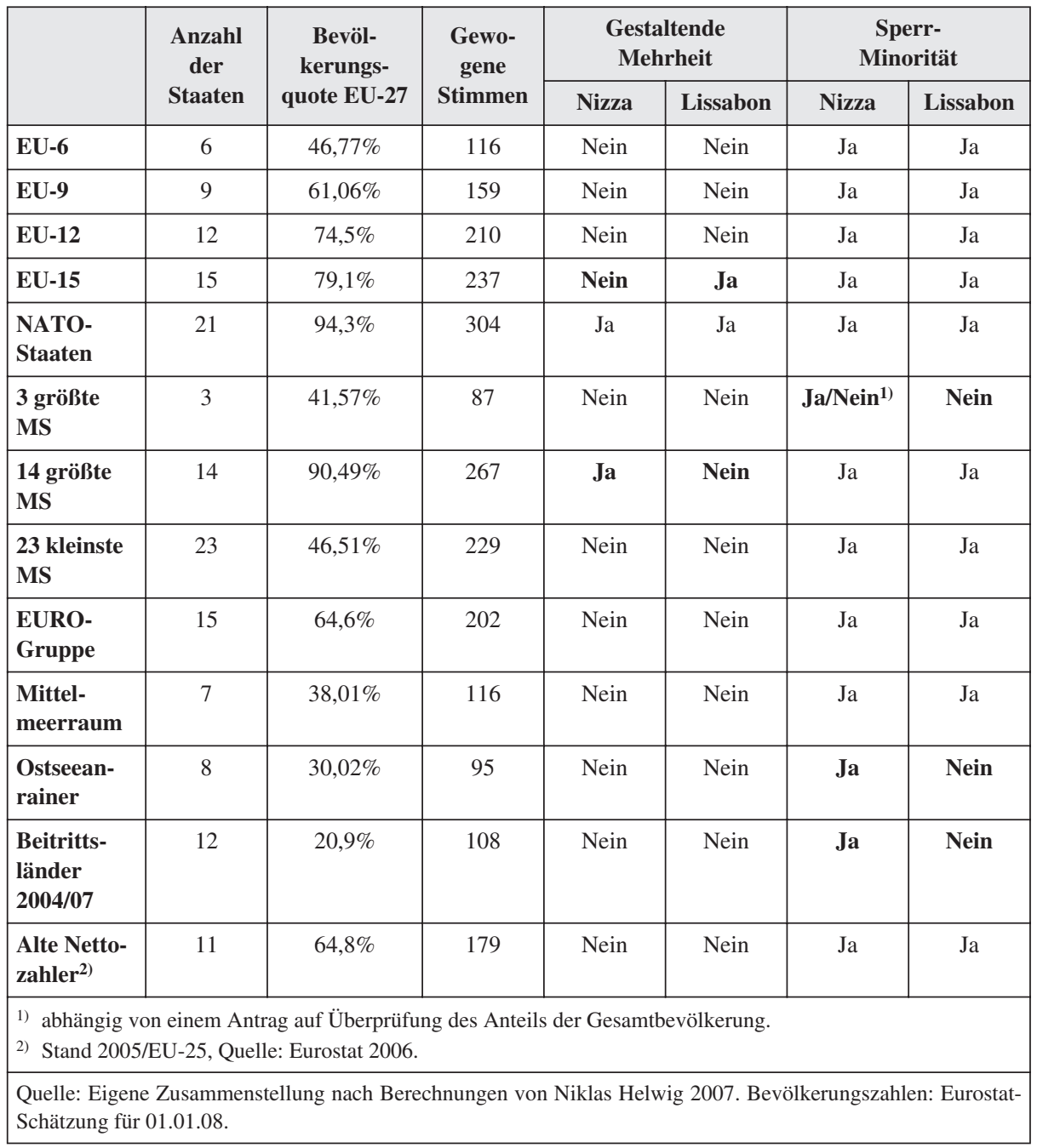

\section{Mehr abgeleitete Demokratie? Der Ausbau der Rechte nationaler Parlamente}

Die möglichen Spannungen im Hinblick auf die Legitimitätsdimension schlagen sich erneut und nachhaltiger als in bisherigen Vertragswerken in der Stärkung der Rolle der nationalen Parlamente nieder (Artikel 12 EUV - siehe Übersicht 6). 


\section{Übersicht 6: Ausbau der Rolle nationaler Parlamente}

- Umfassende Unterrichtung (Artikel 12 EUV)

- Beteiligung an den Mechanismen zur Bewertung der Durchführung der Unionspolitiken im Bereich des Raums der Freiheit, der Sicherheit und des Rechts (Artikel 12 EUV)

- Beteiligung an der Kontrolle der Tätigkeiten von Europol (Artikel 88 AEUV)

- Einspruchsrecht eines einzelnen Parlaments im Rahmen der ,allgemeinen Passerelle“ (Artikel 48 Absatz 7 EUV)

- Einspruchsrecht eines einzelnen Parlaments im Bereich des Familienrechts im Rahmen der justiziellen Zusammenarbeit in Zivilsachen (Artikel 81 Absatz 3 AEUV)

- Einspruchsrecht eines Quorums von nationalen Parlamenten bei Gesetzgebungsakten im Rahmen des Subsidiaritätsprotokolls (Artikel 12 EUV)

Eigene Darstellung.

Neben einem umfassenderen Anspruch auf Unterrichtung wird ihnen ein Satz an Einspruchsrechten zuteil. ${ }^{56}$ Nach entsprechenden Protokollen haben nationale Parlamente im Rahmen eines ,Frühwarnsystems ‘ acht Wochen nach Vorlage eines Entwurfs für einen Gesetzgebungsakt Zeit, eine begründete Stellungnahme hinsichtlich der Übereinstimmung des Entwurfs mit dem Grundsatz der Subsidiarität abzugeben. Übersteigt die Anzahl der negativen Stellungnahmen ein bestimmtes Quorum, muss der Entwurf ,überprüft“ werden. Dieses Quorum beträgt im Falle von Vorlagen beim ordentlichen Gesetzgebungsverfahren die Mehrheit, im Bereich der polizeilichen und justiziellen Zusammenarbeit in Strafsachen ein Viertel, und in allen übrigen Fällen ein Drittel aller Parlamente. ${ }^{57}$ Das Erreichen derartiger Schwellenwerte hat jedoch nur aufschiebende Wirkung. Die für die Vorlage des Entwurfs zuständige Institution - im Regelfall die Kommission - kann nach Prüfung der Einwände beschließen, ,,an dem Entwurf festzuhalten, ihn zu ändern oder ihn zurückzuziehen“. Hält die Kommission bei einem Gesetzgebungsakt im Rahmen des ordentlichen Gesetzgebungsverfahrens an ihrem gerügten Entwurf fest, können der Rat mit einer Mehrheit von 55 Prozent seiner Mitglieder oder das Parlament mit der Mehrheit der abgegebenen Stimmen den Entwurf verwerfen. Die für diese Ablehnung notwendigen Quoren ähneln beziehungsweise überschreiten jedoch ohnehin die Anforderungen an Sperrminoritäten im Rechtsetzungsprozess, sodass bei diesem Regelwerk innerhalb der EU-Architektur keine qualitativ neuen Verfahrensformen eingeführt werden.

Von nachhaltiger Relevanz könnte sich jedoch die Zuständigkeit des Gerichtshofs für die Befolgung des „Protokolls über die Anwendung der Grundsätze der Subsidiarität und der Verhältnismäßigkeit“ erweisen. ${ }^{58}$ Nach dessen Artikel 8 kann eine Klage wegen eines vermeintlichen Verstoßes gegen das Subsidiaritätsprinzip dem Gerichtshof ,,von einem Mitgliedstaat im Namen seines nationalen Parlaments oder einer Kammer dieses Parlaments übermittelt werden“. Der Gerichtshof könnte so zum ,Partner' nationaler Parlamente gegen die qualifizierte Mehrheit nationaler Regierungen und der Abgeordneten des Europäischen Parlaments werden.

56 Protokoll über die Rolle der nationalen Parlamente in der Union und Protokoll über die Anwendung der Grundsätze der Subsidiarität und der Verhältnismäßigkeit.

57 Das „Protokoll über die Anwendung der Grundsätze der Subsidiarität und der Verhältnismäßigkeit“ sieht vor, jedem Parlament zwei Stimmen zuzuteilen. Diese werden in Zweikammersystemen auf beide Kammern verteilt. Die Quoren beziehen sich auf die Zahl der Stimmen - unterschiedliche Kammern können so unterschiedliche Meinungen kundgeben.

58 Vgl. CEPS/EGMONT/EPC: The Treaty of Lisbon, 2007, S. 85-86. 


\section{Eine tragfähige und abschließende Lösung?}

Die Übersicht über die Kompetenz-, die institutionelle und die Verfahrensordnung nach dem Vertrag von Lissabon hat die zentralen Dilemmata der Mitgliedstaaten in ihrem Versuch verdeutlicht, für die gegenwärtigen Gestaltungsprobleme einen adäquaten Problemlösungsraum sowie eine möglichst effiziente Verfahrensordnung unter Wahrung nationalstaatlicher Domänen und legitimatorischer Grundanforderungen zu finden. Das Resultat ist erneut - wie nicht anders zu erwarten - gezeichnet von einer Kompromiss und Konsens ermöglichenden Komplexität.

An diesen Befund knüpft sich die Frage, ob die gefundene Lösung angesichts dieser mangelnden Struktur von Dauer sein kann. ${ }^{59}$ Begegnet der Vertrag von Lissabon abschließend dem dreifachen Dilemma?

Eine Reihe von Veränderungen des Regelwerks lassen bei einzelnen Institutionen und Verfahren ein Mehr an Handlungsfähigkeit sowie demokratischer Mitwirkung und Kontrolle erwarten. $\mathrm{Ob}$ und wie diese Auswirkungen der Neuregelung einander verstärken oder vielleicht sogar beeinträchtigen, wird sich erst im Laufe einer Erprobungsphase herausstellen. Die Akteure werden vertraglich angelegte Spannungsfelder ausloten und gegebenenfalls zu einem neuen institutionellen Gleichgewicht führen, das dann in der Balance der geplanten Steigerung der Handlungsfähigkeit und der beabsichtigten Verbesserung der demokratischen Teilhabe eine neue Stufe im konstatierten Fusionsprozess erlangt.

Es ist hingegen nicht auszuschließen, dass sich die Neuerungen des Lissabonner Vertrags in der Erprobungsphase als nicht tragfähig erweisen und das Dokument - wie die Vertragsrevisionen der letzten zwei Jahrzehnte - schon bald wieder revisionsbedürftig sein könnte. Die Hoffnung der Beteiligten besteht in erster Linie darin, die gegenwärtige Diskussion von institutionellen Fragen, die die Agenda der Vertragsreform im letzten Jahrzehnt dominiert haben, hin zu inhaltlichen Fragen der Politikgestaltung zu lenken. Im Vordergrund steht der Wunsch nach einem „Europa der Projekte“ ${ }^{60}$

Folgt man einem derartigen, der Monnet-Methode zuzuordnenden Schritt, dann ist zu erwarten, dass sich in der Nutzung der angepassten und neu geschaffenen Instrumente der Union langsam ein erneuter Druck zu institutionellen Reformen aufbaut. ${ }^{61}$ Die Suche nach einer Finalität der Integrationskonstruktion durch einen Prozess der, Vertiefung' ist so in eine neue Phase eingetreten, die aber trotz des ,Schwurs auf Beständigkeit' noch keinen Abschluss der Selbstverständigung über das politische System namens Europäische Union bilden dürfte. Auch wenn das „Verfassungskonzept“" vom Europäischen Rat ,,aufgegeben“ wurde, ${ }^{62}$ so können die Staatsund Regierungschefs damit eine weitergehende Debatte über die Finalität der Integrationskonstruktion auf Dauer nicht unterbinden. „Die Funktion des Verfassungstopos“ erweist sich als „zeitlos auch im europäischen Kontext“ ${ }^{63}$ Die Verfassungsdiskussion ${ }^{64}$ der jüngsten Vergangenheit mag so zunächst als ,Eintagsfliege“ erscheinen, die ihr zugrundeliegenden Probleme und Fragen werden allerdings vom Lissabonner Vertrag nicht in abschließender Form beantwortet.

59 Vgl. CEPS/EGMONT/EPC: The Treaty of Lisbon, 2007, S. 145.

60 Vgl. u.a. José Manuel Barroso: Rede vor dem Europäischen Parlament zur Tagung des Europäischen Rates am 15./16. Juni 2006, abrufbar unter: http://ec.europa.eu/commission_barroso/president/pdf/speech_20060614_ de.pdf (letzter Zugriff: 21.12.2007).

61 Wolfgang Wessels/Anne Faber: Vom Verfassungskonvent zurück zur ,Methode Monnet“? Die Entstehung der ,Road map“ zum EU-Reformvertrag unter deutscher Ratspräsidentschaft, in: integration 04/2007, S. 370-381.

62 Europäischer Rat (Brüssel): Mandat für die Regierungskonferenz 2007, Ziffer 1.

63 Müller-Graff: Die Zukunft des europäischen Verfassungstopos, 2007, S. 236.

64 Vgl. Thomas Diez/Antje Wiener: Introducing the Mosaic of Integration Theory, in: Thomas Diez/Antje Wiener (Hrsg.): Theories of European Integration: Past, Present and Future, Oxford 2004, S. 1-21, hier S. 10. 